\title{
Decision making with D-dimer in the diagnosis of pulmonary embolism
}

Wezi Sendama MBBS, MRCP(UK)

Institute of Cellular Medicine, Newcastle University, United Kingdom

Newcastle upon Tyne Hospitals NHS Foundation Trust, Newcastle-upon-Tyne, United Kingdom

Kathryn M Musgrave BA (Hons), MB BS, MRCP(UK)

Institute of Cellular Medicine, Newcastle University, United Kingdom

Newcastle upon Tyne Hospitals NHS Foundation Trust, Newcastle-upon-Tyne, United Kingdom

Corresponding author:

Dr Wezi Sendama

Institute of Cellular Medicine

Newcastle University

$2^{\text {nd }}$ floor William Leech Building

Framlington Place

Newcastle-upon-Tyne

$\mathrm{NE} 24 \mathrm{HH}$

United Kingdom

Email:wezi.sendama@newcastle.ac.uk

Telephone: +(44) 1912085851

This work received no specific grant from any funding agency in the public, commercial, or not-forprofit sectors. Both authors were involved in preparing the manuscript.

Conflicts of interest: None

Article type: Review

Key words: D-dimer; pulmonary embolism; PE; VTE; clinical decision; diagnosis; laboratory test

Running head: D-dimer in the diagnosis of pulmonary embolism 


\section{Decision making with D-dimer in the investigation of pulmonary embolism}

Measurement of a D-dimer level can be a powerful tool in the diagnosis of venous

thromboembolism in the right context and with the right application. In conjunction with estimation of pre-test probability of venous thromboembolism, a D-dimer level can be used to risk stratify patients presenting with clinical features of venous thromboembolism such as pulmonary embolism. Used correctly, a clinical strategy combining pre-test probability estimation and D-dimer measurement can allow safe discharge of patients with suspected pulmonary embolism without superfluous investigation or unnecessary interim treatment.

\section{Where does D-dimer come from?}

Fibrinolysis is a tightly regulated and necessary mechanism to control thrombus propagation and maintain blood fluidity. The activation of coagulation by tissue injury and inflammation results in the deposition of fibrin-containing thrombus, and to avoid excessive thrombus and to allow thrombus to be remodelled and cleared as tissues heal, cross-linked fibrin is broken down by plasmin. ${ }^{1}$ This fibrinolysis results in the release of so-called fibrin degradation products into the circulation. The smallest of these degradation products, D-dimer, is routinely measured in clinical practice as a proxy marker of activation of fibrinolysis. ${ }^{2}$ Fibrinolysis is activated as thrombus is formed, so the D-dimer thereby becomes clinically useful to identify patients in whom pathological intravascular thrombus might be accumulating.

Given that inflammation can activate the coagulation pathway through cytokine-mediated upregulation of endothelial cell surface tissue factor expression, ${ }^{3} \mathrm{D}$-dimer can also be considered a surrogate inflammatory marker. As such, an elevated D-dimer laboratory test cannot be considered a specific marker of venous thromboembolism, but its sensitivity makes it an asset in the diagnosis 
of venous thromboembolism such as pulmonary embolism where the pre-test probability of venous thromboembolism has been appropriately assessed. ${ }^{4}$

\section{$\underline{\text { D-dimer as a diagnostic test for pulmonary embolism }}$}

The contemporary clinical use of D-dimer in the investigation of pulmonary embolism can probably be attributed to the efforts of Philip Wells and colleagues. Wells' work with combining a clinical scoring algorithm and the laboratory D-dimer test to estimate the probability of pulmonary embolism was borne of the limitations of ventilation-perfusion lung scanning, which was the practical diagnostic standard for pulmonary embolism at the turn of the $21^{\text {st }}$ century. ${ }^{5}$ Ventilationperfusion scans were occasionally non-diagnostic for pulmonary embolism, and if clinical suspicion for pulmonary embolism was high despite a non-diagnostic ventilation-perfusion scan and equivocal lower limb ultrasound for deep venous thrombosis, patients would sometimes subsequently be subjected to invasive pulmonary angiography and its associated risks. ${ }^{6}$

The increasing utility of computed tomography pulmonary angiography in the intervening decade made the question of which patients to recommend for invasive angiography less relevant, but the use of ionising radiation and intravascular contrast media to confirm the diagnosis with computed tomography means that estimation of probability of pulmonary embolism before deciding whether to request computed tomography remains imperative. To this end the work of Wells and others is highly relevant, although several authors report inadequate estimation of probability of pulmonary embolism prior to computed tomography resulting in an overuse of computed tomography. ${ }^{7-9}$

The utility of the D-dimer in the diagnosis of pulmonary embolism is maximised when the pre-test probability of pulmonary embolism is estimated. The Wells score emerged from a study of consecutive adult inpatients and outpatients with suspected pulmonary embolism with the aim of simplifying the estimation of pre-test probability (Table 1). Using prior observations of the varying likelihood of pulmonary embolism diagnosis with particular clinical features, Wells et al. constructed a clinical model that was first used to justify withholding anticoagulation where ventilation-perfusion 
scanning had proved inconclusive but pre-test probability had been low, and then later to justify ruling out pulmonary embolism where pre-test probability was acceptably low and D-dimer was negative. $^{10,11}$

As well as identifying features strongly associated with pulmonary embolism (such as tachycardia, haemoptysis and clinical features of lower limb venous thromboembolism), part of the assessment of pre-test probability of pulmonary embolism typically includes a clinician's subjective assessment as to whether the clinical features identified in the patient are most likely to be attributed to pulmonary embolism. Wells and colleagues' work to derive a clinical decision rule for diagnosis of pulmonary embolism suggested that where a clinician felt the observed clinical features were unlikely to be explained by a different diagnosis, the likelihood of subsequent confirmation of pulmonary embolism was increased four- to sixfold. ${ }^{11}$ (This is the basis of "alternative diagnosis less likely than pulmonary embolism" carrying 3 points on the Wells score for pulmonary embolism.)

A clinical conundrum in the medical assessment unit can arise when a patient is assessed with knowledge of the D-dimer result, as can sometimes happen when blood tests are ordered before clinical assessment in the emergency department in an attempt to improve patient flow. ${ }^{12}$ Prior knowledge of the laboratory result can influence the clinician's subjective assessment of whether an alternative diagnosis is less likely than pulmonary embolism and may therefore result in computed tomography scanning and/or anticoagulation where pulmonary embolism is unlikely, or no attempt to confirm a diagnosis of pulmonary embolism where it might be likely. ${ }^{13-15}$ It is possible, then, that strategies to minimise this bias might reduce patient harms caused by inappropriate investigation or treatment.

\section{Negative D-dimer at point of assessment}

Although the D-dimer assays in common use are sensitive for the detection of intravascular thrombus formation (a systematic review by Di Nisio et al. suggests $95 \%$ sensitivity of microplate enzyme-linked immunosorbent D-dimer assays for the detection of PE), a negative D-dimer does not 
necessarily rule out pulmonary embolism on its own. ${ }^{16}$ Wells and colleagues' retrospective analysis of a cohort of almost 1000 patients investigated for pulmonary embolism suggested a $3 \%$ rate of pulmonary embolism in those with a negative D-dimer regardless of pre-test probability. In the subgroup of patients in whom the pre-test probability of pulmonary embolism was deemed high (Wells score $>4$ ) but D-dimer was negative, the rate of pulmonary embolism was $16 \% \cdot{ }^{11}$ Gibson et al. demonstrated a similar false-negative rate: a $2 \%$ rate of pulmonary embolism was observed in all patients suspected of pulmonary embolism with a negative D-dimer, and this rose to $9 \%$ in the subgroup with Wells score $>4$ and negative $\mathrm{D}$-dimer. ${ }^{13}$

Given the potentially grave consequences of missing a pulmonary embolism diagnosis having been reassured by a negative D-dimer result, a clinician assessing a patient for suspected pulmonary embolism where the D-dimer is already known to be negative should ensure that an attempt at pretest probability scoring is made using a clinical decision rule such as the Wells score. A score that suggests a high likelihood of pulmonary embolism should always prompt further investigation regardless of the D-dimer result. On the other hand, a Wells score that suggests a low likelihood of pulmonary embolism (less than or equal to 4 ) in conjunction with a negative D-dimer was associated with a $2 \%$ rate of pulmonary embolism in Wells' original derivation cohort, but a later meta-analysis of prospective studies suggested a pooled incidence of pulmonary embolism of $0.34 \%$ in such populations. ${ }^{11,17}$ This would probably give most clinicians reassurance that pulmonary embolism had been ruled out.

\section{$\underline{\text { Positive D-dimer at point of assessment }}$}

As above, the approach to the assessment of a patient with suspected pulmonary embolism known to have a positive D-dimer should begin with an estimation of the pre-test probability of pulmonary embolism, ideally using a clinical decision rule. However, given that three points of the Wells score hinge on the clinician's subjective assessment of whether pulmonary embolism is the most likely explanation of the clinical observations, it is possible that knowledge of a positive D-dimer result 
could bias the clinician in favour of pulmonary embolism. This could lead to unnecessary subsequent investigation or even treatment with anticoagulation in an effort to keep the patient "safe" until diagnostic imaging can be performed.

A questionnaire-based study undertaken in the Netherlands found that when physicians were presented with hypothetical cases with high diagnostic uncertainty for pulmonary embolism, the inclusion of an abnormal D-dimer result in the clinical information increased the probability of a clinician assigning a Wells score greater than 4 (which is the cut-off for "pulmonary embolism likely" on the two-level Wells score).${ }^{18} \mathrm{~A}$ strategy to combat this bias might be to avoid looking at a D-dimer result before the clinical assessment if the temptation can be resisted, but the success of such a technique might depend upon the limitations of human nature. A more acceptable strategy might be to estimate the pre-test probability of pulmonary embolism retrospectively using a clinical decision rule that does not incorporate a subjective aspect, such as the revised Geneva score (Table 2). ${ }^{19}$ Using the revised Geneva score, a score of 0-3 indicates a low clinical probability of pulmonary embolism, and in the original validation data this corresponded with a $7.9 \%$ prevalence of pulmonary embolism (95\% confidence interval 5.0-12.1\%). ${ }^{19}$ In this low probability group an elevated D-dimer is likely to be a false positive result, given the poor specificity of commonly used Ddimer assays for pulmonary embolism. ${ }^{16}$ Scores of 4 and above, however, should warrant further investigation where a D-dimer result is already known to be positive.

Meta-analyses have suggested that the Wells score stratifies pre-test probability of pulmonary embolism with similar accuracy to the revised Geneva score ${ }^{20,21}$ although there has not yet been a comparison of the two clinical decision scores in scenarios where the clinician has prior knowledge of the D-dimer result. As such, it is difficult to know whether using the revised Geneva score rather than the Wells score would truly eliminate the potential bias introduced by knowledge of the Ddimer result prior to clinical assessment. 
The concept of "inflamm-aging" was borne of the observation that increasing age is associated with increasing levels of chronic low-grade systemic inflammation due to dysregulated immune responses. ${ }^{22}$ With increasing levels of systemic inflammation higher levels of soluble inflammatory markers can be measured in the blood, and it has also been demonstrated that higher baseline levels of D-dimer can be measured with increasing age. ${ }^{23,24}$ This may reflect the ability of D-dimer to act as a proxy inflammatory marker as discussed previously in this article.

Given apparently higher baseline levels of D-dimer with age, the clinical utility of a fixed upper limit of normal (traditionally 500 $\mathrm{\mu g} / \mathrm{L}$ ) has been called into question. Previous studies have suggested a reduction in the specificity of D-dimer for venous thromboembolism detection in older patients using a fixed cut-off value, and a recovery of specificity without loss of sensitivity by increasing the cut-off value with age. ${ }^{23,25}$ By retrospectively analysing previous data from cohort studies evaluating the diagnosis of pulmonary embolism, Douma et al. postulated that in patients older than 50 , the clinical utility of the D-dimer test to detect pulmonary embolism increased if the cut-off was taken to be (age $\times 10) \mu \mathrm{g} / \mathrm{L.}^{25}$

Following this work, a prospective study was undertaken to assess the utility and safety of an ageadjusted cut-off value for D-dimer in the diagnosis of pulmonary embolism. The ADJUST-PE study was a multicentre study of 3324 patients with suspected pulmonary embolism from hospitals in Belgium, France, the Netherlands and Switzerland in which the primary outcome measure was the failure rate of a diagnostic strategy using a clinical decision rule and a D-dimer cut-off value of (age $\times 10) \mu g / L$ in patients older than 50 . Patients younger than 50 years of age were evaluated using the traditional cut-off of $500 \mu \mathrm{g} / \mathrm{L}$. Although the study was not designed to compare the new strategy against usual practice directly, the failure rates of the new strategy were acceptably low, suggesting it to be safe. In addition, the age-adjusted D-dimer proved particularly useful in ruling out pulmonary embolism in patients aged 75 or older without further investigation: it was suggested that where 1 in 16 patients would have had PE ruled out using the $500 \mu \mathrm{g} / \mathrm{L}$ cut-off, this increased to 1 in 3.4 using 
the age-adjusted cut-off. ${ }^{26}$ This would indicate potential for cost savings by adopting the use of ageadjusted cut-offs.

Limitations of the use of D-dimer in pulmonary embolism diagnosis

Even aside from the relative lack of specificity of D-dimer as discussed above, diagnosis of pulmonary embolism using D-dimer remains liable to pitfalls. Pregnant women have been notable absentees from the studies that inform contemporary strategies to diagnose pulmonary embolism. This is despite pregnancy conferring a seven- to tenfold increase in the risk of venous thromboembolism compared to age-matched controls. ${ }^{27}$ The use of D-dimer to aid pulmonary embolism diagnosis in pregnant women is not typically recommended in part due to observations of rising baseline levels of D-dimer in pregnant women over the course of pregnancy, which might reduce the specificity of a fixed threshold value for pulmonary embolism detection. ${ }^{28}$ In any case, there remains a dearth of prospective studies aimed at formulating a clinical decision strategy for pulmonary embolism diagnosis in pregnant women.

The DiPEP study was a prospective cohort study of 324 pregnant and postpartum women in the United Kingdom that attempted to assess whether clinical features or D-dimer (alone or in combination) could be used to rule out pulmonary embolism without further diagnostic imaging. 198 cases of confirmed pulmonary embolism were also analysed retrospectively to enable more precise estimates of sensitivity. The investigators found that existing clinical decision rules for pulmonary embolism were poor indicators of which patients were subsequently diagnosed with pulmonary embolism, and D-dimer thresholds (whether fixed at 500 $\mathrm{g} / \mathrm{L}$ or adjusted for trimester) did not have sufficient discriminatory value for the test to be useful in diagnosis. ${ }^{29}$ For the gestation-adjusted Ddimer thresholds, the DiPEP investigators used three discrete D-dimer threshold values-a threshold for each trimester-rather than the construction of a threshold varying continuously with gestational age as in the ADJUST-PE study. This might have cost the D-dimer some of its value as a 
diagnostic tool in this study, although it may well be that $D$-dimer simply has no diagnostic value for pulmonary embolism detection in pregnancy.

In considering the value of D-dimer to exclude venous thromboembolism, causes of false-normal Ddimer should also be considered. A readily apparent cause is concurrent use of anticoagulation, which would reduce the chance of fibrin being formed by the coagulation cascade and would therefore result in the observation of fewer degradation products from fibrinolysis. ${ }^{1}$

\section{Advances in biomarkers for venous thromboembolism}

Given the low specificity of the D-dimer test for venous thromboembolism detection, the diagnostician's dream would probably be the development of a new test that would quickly return a positive result in every case of venous thromboembolism without a risk of false positives. Although that remains far-fetched at present, research taking advantage of high-throughput proteomics may be able to offer some hope of improving upon current laboratory tests for venous thromboembolism.

By analysing the protein contents of blood samples from recently-diagnosed venous thromboembolism patients and controls, the investigators of the VEREMA study were able to identify strong associations between venous thromboembolism and plasma levels of four proteins: human immunodeficiency virus type I enhancer binding protein 1, von Willebrand factor and platelet-derived growth factor $\beta$. The associations of von Willebrand factor and platelet-derived growth factor $\beta$ with venous thromboembolism were observed once more when the investigators replicated the findings by profiling an external biobank, leading to the conclusion that plateletderived growth factor $\beta$ could have merit as a biomarker for venous thromboembolism risk. ${ }^{30}$ Menon et al. used similar techniques to identify plasma proteins strongly associated with the diagnosis of venous thromboembolism and then attempted to assess the diagnostic potential of combinations of these proteins in the detection of venous thromboembolism. A combination of 
activated protein C-protein C inhibitor and osteopontin demonstrated comparable sensitivity to Ddimer and higher specificity when tested retrospectively on blood samples from patients diagnosed with venous thromboembolism and controls. ${ }^{31}$

Despite showing promise, experimental correlations between venous thromboembolism and elevated levels of plasma proteins might not be sufficient to supplant established laboratory tests such as D-dimer without further investigation. Nevertheless, such work suggests that improvements on currently available diagnostics may be on the horizon.

\section{Conclusions}

D-dimer, despite its flaws, remains a valuable tool in the diagnosis of venous thromboembolism. Although it may be increasingly common to have a D-dimer result available before clinical assessment, that should not preclude a structured approach to pulmonary embolism diagnosis, and considered use of available information should minimise the number of patients inappropriately investigated or treated. Further work remains to be done on eliminating diagnostic error arising from knowledge of the D-dimer result prior to clinical assessment, and future work should also attempt to clarify the uncertain role of D-dimer in venous thromboembolism diagnosis in pregnancy. It remains to be seen whether recent developments in high-throughput proteomics can result in biomarkers that might supplant D-dimer in the diagnosis of venous thromboembolism.

\section{Acknowledgements}

This work received no specific grant from any funding agency in the public, commercial, or not-forprofit sectors.

\section{References}

1. Cesarman-Maus G, Hajjar KA. Molecular mechanisms of fibrinolysis. Br J Haematol. 2005;129(3):307-321. doi:10.1111/j.1365-2141.2005.05444.x

2. Tripodi A. D-dimer testing in laboratory practice. Clin Chem. 2011;57(9):1256-1262. 
doi:10.1373/clinchem.2011.166249

3. Petäjä J. Inflammation and coagulation. An overview. Thromb Res. 2011;127:S34-S37. doi:10.1016/S0049-3848(10)70153-5

4. Ginsberg JS, Wells PS, Kearon C, et al. Sensitivity and Specificity of a Rapid Whole-Blood Assay for D-Dimer in the Diagnosis of Pulmonary Embolism. Ann Intern Med. 1998;129(12):1006. doi:10.7326/0003-4819-129-12-199812150-00003

5. Wells PS, Anderson DR, Rodger M, et al. Excluding Pulmonary Embolism at the Bedside without Diagnostic Imaging: Management of Patients with Suspected Pulmonary Embolism Presenting to the Emergency Department by Using a Simple Clinical Model and d-dimer. Ann Intern Med. 2001;135(2):98. doi:10.7326/0003-4819-135-2-200107170-00010

6. Rathbun SW, Raskob GE, Whitsett TL. Sensitivity and Specificity of Helical Computed Tomography in the Diagnosis of Pulmonary Embolism. Ann Intern Med. 2000;132(3):227. doi:10.7326/0003-4819-132-3-200002010-00009

7. Perelas A, Dimou A, Saenz A, et al. CT Pulmonary Angiography Utilization in the Emergency Department. Am J Med Qual. 2015;30(6):571-577. doi:10.1177/1062860614543302

8. Crichlow A, Cuker A, Mills AM. Overuse of Computed Tomography Pulmonary Angiography in the Evaluation of Patients with Suspected Pulmonary Embolism in the Emergency Department. Acad Emerg Med. 2012;19(11):1219-1226. doi:10.1111/acem.12012

9. van der Hulle T, Cheung WY, Kooij S, et al. Simplified diagnostic management of suspected pulmonary embolism (the YEARS study): a prospective, multicentre, cohort study. Lancet. 2017;390(10091):289-297. doi:10.1016/S0140-6736(17)30885-1

10. Wells PS, Ginsberg JS, Anderson DR, et al. Use of a Clinical Model for Safe Management of Patients with Suspected Pulmonary Embolism. Ann Intern Med. 1998;129(12):997. doi:10.7326/0003-4819-129-12-199812150-00002 
11. Wells PS, Anderson DR, Rodger M, et al. Derivation of a simple clinical model to categorize patients probability of pulmonary embolism: increasing the models utility with the SimpliRED D-dimer. Thromb Haemost. 2000;83(3):416-420.

12. Jones $\mathrm{P}$, Elangbam B, Williams NR. Inappropriate use and interpretation of D-dimer testing in the emergency department: an unexpected adverse effect of meeting the "4-h target". Emerg Med J. 2010;27(1):43-47. doi:10.1136/emj.2009.075838

13. Gibson NS, Sohne M, Gerdes VEA, Nijkeuter M, Buller HR. The Importance of Clinical Probability Assessment in Interpreting a Normal d-Dimer in Patients With Suspected Pulmonary Embolism. Chest. 2008;134(4):789-793. doi:10.1378/CHEST.08-0344

14. Kristoffersen AH, Ajzner E, Rogic D, et al. Is D-dimer used according to clinical algorithms in the diagnostic work-up of patients with suspicion of venous thromboembolism? A study in six European countries. Thromb Res. 2016;142:1-7. doi:10.1016/j.thromres.2016.04.001

15. Douma RA, Kessels JBF, Buller HR, Gerdes VEA. Knowledge of the D-dimer test result influences clinical probability assessment of pulmonary embolism. Thromb Res. 2010;126(4):e271-e275. doi:10.1016/J.THROMRES.2010.07.008

16. Di Nisio M, Squizzato A, Rutjes AWS, Büller HR, Zwinderman AH, Bossuyt PMM. Diagnostic accuracy of D-dimer test for exclusion of venous thromboembolism: a systematic review. J Thromb Haemost. 2007;5(2):296-304. doi:10.1111/j.1538-7836.2007.02328.x

17. Pasha SM, Klok FA, Snoep JD, et al. Safety of excluding acute pulmonary embolism based on an unlikely clinical probability by the Wells rule and normal D-dimer concentration: A metaanalysis. Thromb Res. 2010;125(4):e123-e127. doi:10.1016/J.THROMRES.2009.11.009

18. Gibson N, Douma R, Squizzato A, Sohne M, Buller H, Gerdes V. Application of a decision rule and a D-dimer assay in the diagnosis of pulmonary embolism. Thromb Haemost. 2010;103(04):849-854. doi:10.1160/TH08-09-0564 
19. Le Gal G, Righini M, Roy P-M, et al. Prediction of Pulmonary Embolism in the Emergency Department: The Revised Geneva Score. Ann Intern Med. 2006;144(3):165. doi:10.7326/0003-4819-144-3-200602070-00004

20. Ceriani E, Combescure C, Le Gal G, et al. Clinical prediction rules for pulmonary embolism: a systematic review and meta-analysis. J Thromb Haemost. 2010;8(5):957-970. doi:10.1111/j.1538-7836.2010.03801.x

21. Lucassen W, Geersing G-J, Erkens PMG, et al. Clinical Decision Rules for Excluding Pulmonary Embolism: A Meta-analysis. Ann Intern Med. 2011;155(7):448. doi:10.7326/0003-4819-155-7201110040-00007

22. Pawelec G, Goldeck D, Derhovanessian E. Inflammation, ageing and chronic disease. Curr Opin Immunol. 2014;29:23-28. doi:10.1016/J.COI.2014.03.007

23. Harper PL, Theakston E, Ahmed J, Ockelford P. D-dimer concentration increases with age reducing the clinical value of the D-dimer assay in the elderly. Intern Med J. 2007;37(9):607613. doi:10.1111/j.1445-5994.2007.01388.x

24. Haase C, Joergensen M, Ellervik C, Joergensen MK, Bathum L. Age- and sex-dependent reference intervals for D-dimer: Evidence for a marked increase by age. Thromb Res. 2013;132(6):676-680. doi:10.1016/J.THROMRES.2013.09.033

25. Douma RA, le Gal G, Söhne M, et al. Potential of an age adjusted D-dimer cut-off value to improve the exclusion of pulmonary embolism in older patients: a retrospective analysis of three large cohorts. BMJ. 2010;340:c1475. doi:10.1136/BMJ.C1475

26. Righini M, Van Es J, Den Exter PL, et al. Age-Adjusted D-Dimer Cutoff Levels to Rule Out Pulmonary Embolism: The ADJUST-PE Study. JAMA. 2014;311(11):1117. doi:10.1001/jama.2014.2135

27. Bourjeily G, Paidas M, Khalil H, Rosene-Montella K, Rodger M. Pulmonary embolism in 
pregnancy. Lancet. 2009;375:500-512. doi:10.1016/S0140

28. Van der Pol LM, Mairuhu ATA, Tromeur C, Couturaud F, Huisman MV, Klok FA. Use of clinical prediction rules and D-dimer tests in the diagnostic management of pregnant patients with suspected acute pulmonary embolism. Blood Rev. 2017;31(2):31-36.

doi:10.1016/J.BLRE.2016.09.003

29. Goodacre S, Horspool K, Nelson-Piercy C, et al. The DiPEP study: an observational study of the diagnostic accuracy of clinical assessment, D-dimer and chest x-ray for suspected pulmonary embolism in pregnancy and postpartum. BJOG An Int J Obstet Gynaecol. June 2018. doi:10.1111/1471-0528.15286

30. Bruzelius $\mathrm{M}$, Iglesias MJ, Hong M-G, et al. PDGFB, a new candidate plasma biomarker for venous thromboembolism: results from the VEREMA affinity proteomics study. Blood. 2016;128(23):e59-e66. doi:10.1182/blood-2016-05-711846

31. Memon AA, Sundquist K, PirouziFard M, et al. Identification of novel diagnostic biomarkers for deep venous thrombosis. Br J Haematol. 2018;181(3):378-385. doi:10.1111/bjh.15206 


\begin{tabular}{l|l} 
Variable & Points \\
\hline Clinical signs and symptoms of deep vein thrombosis & 3.0 \\
Immobilisation or surgery in previous 4 weeks & 1.5 \\
Heart rate greater than 100 beats/min & 1.5 \\
Previous history of pulmonary embolism or deep vein thrombosis & 1.5 \\
Haemoptysis & 1.0 \\
\hline Malignancy & \\
\hline Alternative diagnosis is less likely than pulmonary embolism & 3.0
\end{tabular}

Table 1. 2-level Wells score. ${ }^{11}$ If score $\leq 4$ pulmonary embolism unlikely; if score $>4$ pulmonary embolism likely 


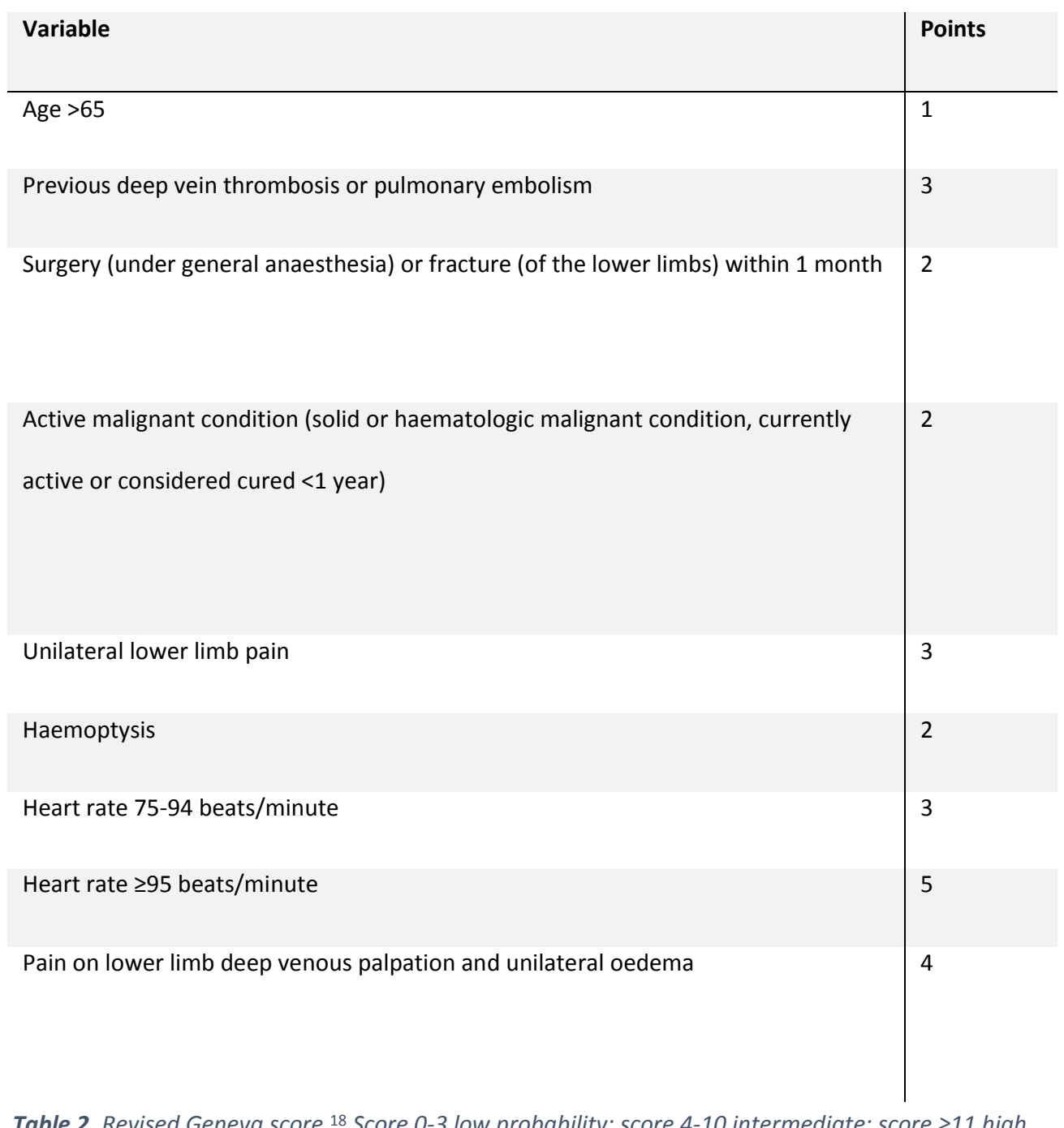

Table 2. Revised Geneva score. ${ }^{18}$ Score 0-3 low probability; score 4-10 intermediate; score $\geq 11$ high 